\title{
Clinical Facial Study of the Parents of Children with OAV (Oculo-Auriculo-Vertebral) Syndrome. Cases and Controls
}

\author{
María de la Luz Arenas-Sordo ${ }^{*}$, Claudia I. Cruz-Ugalde1, Eny Paola Linares-Mendoza1, \\ Julieta Karina Peñuelas-Romero', Beatriz C. Aldape-Barrios ${ }^{2}$ \\ ${ }^{1}$ Genetics Department, Instituto Nacional de Rehabilitación “Luis Guillermo Ibarra Ibarra”, Mexico City, México \\ ${ }^{2}$ School of Dentistry, Universidad Nacional Autónoma de México, University City, Mexico City, México \\ Email: ^mlarenassordo@hotmail.com, mlarenassordo@gmail.com, marenas@inr.gob.mx, asgk@unam.mx, \\ kallana2001@gmail.com,linares.paola@gmail.com,karipenuelas@hotmail.com,patobu1@unam.mx
}

How to cite this paper: Arenas-Sordo, M.L., Cruz-Ugalde, C.I., Linares-Mendoza, E.P., Peñuelas-Romero, J.K. and Aldape-Barrios, B.C. (2020) Clinical Facial Study of the Parents of Children with OAV (Oculo-Auriculo-Vertebral) Syndrome. Cases and Controls. International Journal of Otolaryngology and Head \& Neck Surgery, 9, 53-60.

https://doi.org/10.4236/ijohns.2020.92008

Received: January 7, 2020

Accepted: February 15, 2020

Published: February 18, 2020

Copyright $\odot 2020$ by author(s) and Scientific Research Publishing Inc. This work is licensed under the Creative Commons Attribution International License (CC BY 4.0).

http://creativecommons.org/licenses/by/4.0/

\begin{abstract}
Introduction: The oculo-auriculo-vertebral syndrome is a complex disease of the head and neck. Aim: The purpose of this study was to look for facial asymmetry, measuring both sides of the mandible and external ear in the parents of these patients in a clinical way, to see if it could be considered as a minor manifestation of the disease. Patients and Methods: A clinical, observational study was done with 50 trios and their parents' controls, at the Instituto Nacional de Rehabilitación LGII in 2019, from January to May. Mandible and ear measurements were done. Results: All of our patients had microtia and facial asymmetry, and in 17 cases, other anomalies. We compared both sides of the mandible in the patients' parents and in the parents' controls. The results showed differences only in the fathers, and in the comparison with the controls, we found statistical differences only in women, family and non-family cases. The ears did not show any differences. Discussion: It is very interesting that we found differences in the measurements of the mandible of the patients' mothers and their controls; in the mothers the mandible was bigger than in the controls, this probably means that the development of the cranial structures is modified by some genetic variants. No differences were found in the measurements of the ears. Conclusion: It is very difficult to know exactly if the differences we found are important or not. The clinical measurements could be a good option to establish if the parents have minor manifestations that could explain some heritability.
\end{abstract}

\section{Keywords}

Oculo-Auriculo-Vertebral Syndrome, Microtia, Facial Asymmetry 


\section{Introduction}

The oculo-auriculo-vertebral or facio-auriculo-vertebral syndrome (OAV/FAV), is a complex disease that involves the embryological development of head and neck dependent on the first and second branchial or pharyngeal arches and with a wide spectrum of anomalies [1].

The frequency of this entity was calculated by Poswillo around 1/4000 LNB in 1973 [2]; after him other frequencies have been mentioned in different populations: Grabb 1/5600, Stoll 1/19,500, Melnick 1/26,500 [3] [4]. The prevalence is considered to be higher in Hispanics, Asians, Native Americans, and Andeans [5]. From the clinical findings, the microtia is the cardinal manifestation but with a lot of other malformations [1] [6]. Another characteristic that is very common is the hemifacial microsomia; this feature can be unnoticed or be very severe, in the latter cases the patients can suffer from chewing and respiratory problems [7].

Beleza A et al. in 2014 mentioned that the multisystemic affectation is not frequent, present only in $10 \%$ of the cases; however we found in a previous study, other malformations besides microtia and hemifacial microsomia, in $38.25 \%$ [8] [9].

It is also mentioned that this pathology is more frequent in males than females, but we did not find it in this report or in the previous one [9].

It is important not to forget that tags and pits are minor manifestations of the disease and it is necessary to look for them in all the family, both sides, paternal and maternal, to establish the diagnosis of familial or non-familial cases [4].

From the hereditary point of view the OAV syndrome is considered heterogeneous; it could be multifactorial, probably Mendelian in some cases or it could be secondary to some chromosome alterations. It is also important to mention that it has been related to some teratogens [1].

There are some genes that are considered candidates because of their action, especially in some embryologic phases of the ear formation [10] (Table 1).

The purpose of this study was to look for facial asymmetry, measuring both sides of the mandible and external ear in the parents of these patients in a clinical way, to see if it could be considered as a minor manifestation of the disease.

To our knowledge, there are no similar studies; in some cases the measurements have been done with panoramic radiographic images or with tridimensional CT-study [11] [12].

\section{Patients and Methods}

A clinical, observational study was done with 50 trios (the patients and their parents) and their parents' controls at the Instituto Nacional de Rehabilitación Luis Guillermo Ibarra Ibarra (INR-LGII) in 2019, from January to May. We invited all patients with OAV syndrome, any sex or age, who would like to participate and with the possibility of measuring their two parents. The patients' parents signed the informed consent. The clinical characteristics we studied were: 
Table 1. Candidate gene.

\begin{tabular}{cc}
\hline Gene & Function \\
\hline MYT 1 & Myelin transcription factor 1 \\
FOXG1 & Transcription factor \\
FAAH2 & fatty acid amide hydrolase 2 \\
DIP2C & disco interacting protein 2 homolog C \\
SIX1/4 & SIX homeobox 1/4 \\
HOXA1 & homeobox A1. Transcription factor \\
HOXA2 & homeobox A2. Transcription factor \\
TBX1 & T-box 1. Transcription factor involved in the \\
SALL1 & regulation of developmental processes \\
TCOF1 & Transcription factor \\
& Craneofacial developmental \\
\hline
\end{tabular}

measurements of the mandible (right and left side), both ears, presence or not of preauricular tags, epibulbar dermoid, microtia (Hunter classification) in patients and parents. In patients we also did CT of the ear and mastoids, renal ultrasonography and complete column $\mathrm{x}$ rays as well as complete clinical exploration and a pedigree with at least three generations.

To carry out the mandible measurements, the distance from the angle to the symphysis was taken. With the ears we took the distance between the upper outer edge of the helix of the ear and the lower edge of the lobe of the ear. Both measurements were done by the same person $($ Kappa $=0.89)$ and with the same vernier tool.

\section{Statistical Analysis}

Mean, mode and SD in quantitative variables, frequencies in qualitative variables, kappa index and T-student for comparison between cases and controls and the size of both sides of the mandible and ears in patients' parents and their controls.

All patients, parents and their controls were from the Central region of Mexico, and were matched by age and sex.

The patients and controls of the parents agreed to participate and signed the informed consent. The project was authorized by the INR-LGII Research and Ethics Committees.

\section{Results}

From de 50 patients studied, $22(44 \%)$ were females and 28 (56\%) males, the mean age was 7 years, with SD 3.6 years and range 1 - 19 years. Almost half (46\%) of the patients have family backgrounds of the same disease. All of the patients had microtia and facial asymmetry, some of them also had other abnormalities (17 cases, 34\%), renal and vertebral anomalies and global developmental 
delay. In Table 2 we show the general demographic and clinical data of the patients. We compared both sides of the mandible in the patients' parents and in the parents' controls. The results showed differences only in the fathers, with $p=$ 0.0253 (Table 3).

In the comparison between the parents of the patients and their controls, we found statistical differences only in women, in family cases as well as in non-family cases (Table 4 ).

When compared both sides of the mandible in the controls (female or male), we found no differences (Table 5).

We also studied the measurements of the patients' parents and their controls, looking for the mean, the standard deviation and the ranges (Table 6).

\section{Discussion}

The OAV syndrome is a clinical genetic disorder with great heterogeneity, which means that it is very complex to study, we can see that there are a lot of clinical findings and differences between populations. In all the Mexican studies [9]

Table 2. Demographic and clinical data of the patients.

\begin{tabular}{|c|c|}
\hline \multicolumn{2}{|l|}{ Age } \\
\hline Range & $1-19$ yrs \\
\hline Mean & $7 \mathrm{yrs}$ \\
\hline SD & $3.6 \mathrm{yrs}$ \\
\hline Sex & $22(44 \%)$ \\
\hline Females/Males & $28(56 \%)$ \\
\hline \multicolumn{2}{|l|}{ Family backgrounds } \\
\hline Yes & $23(46 \%)$ \\
\hline Non & $27(54 \%)$ \\
\hline \multicolumn{2}{|l|}{ Clinical characteristics } \\
\hline Microtia & $50(100 \%)$ \\
\hline Unilateral & $35(70 \%)$ \\
\hline Right side & $29(58 \%)$ \\
\hline Hemifacial microsomia & $50(100 \%)$ \\
\hline Calyceal Dilatation & $1(2 \%)$ \\
\hline Global developmental delay & $2(4 \%)$ \\
\hline Skeletal & $7(14 \%)$ \\
\hline Vertebral fusion anomalies & $2(4 \%)$ \\
\hline Scoliosis & $3(6 \%)$ \\
\hline Vertebral segmentation defect & $1(2 \%)$ \\
\hline Transverse limb defects (upper limb) & $1(2 \%)$ \\
\hline
\end{tabular}


Table 3. Comparison of the two sides of the mandible bone in parent's cases and in controls.

\begin{tabular}{cc}
\hline & Valor de $\mathrm{p}$ \\
\hline RSM vs LSM & 0.8934 \\
RSF vs LSF & $0.0253^{*}$ \\
RSFC vs LSFC & 0.1721 \\
RSMC vs LSMC & 0.2069 \\
\hline
\end{tabular}

RSM = right side, mother; LSM = Left side, mother; $\mathrm{RSF}=$ right side, father; $\mathrm{LSF}=$ Left side, father; $\mathrm{RSFC}=$ right side, female control; LSFC = left side, female control; RSMC = right side, men control; LSMC = left side, men control.

Table 4. Comparison of the two sides of the mandible bone between parents' patients and control in family and in non-family cases.

\begin{tabular}{cc}
\hline Family cases & $\mathrm{p}$ \\
\hline RSM vs RSFC & $0.0062^{\star}$ \\
LSM vs LSC & $0.0037^{*}$ \\
RSF vs RSMC & 0.2933 \\
LSF vs LSMC & 0.2651 \\
\hline Non-family cases & $0.0007^{*}$ \\
\hline RSM vs RSFC & $0.0032^{*}$ \\
LSM vs LSC & 0.0961 \\
RSF vs RSMC & 0.692 \\
\hline LSF vs LSMC & $1 S F C=1$ side \\
\hline
\end{tabular}

RSM $=$ right side mother, RSC $=$ right side female control, $\mathrm{LSM}=$ left side mother, $\mathrm{LSFC}=$ left side female control, RSF $=$ right side father, $\mathrm{RSMC}=$ right side male control, $\mathrm{LSF}=$ left side father, $\mathrm{LSMC}=$ left side male control.

Table 5. Measurements of the mandible of the parents of the patients and of the controls of the parents.

\begin{tabular}{cc}
\hline Parents/Controls & Mean $(\mathrm{SD}) /$ ranges \\
\hline RMM & $9.9254(0.8109) / 8.5-12.4$ \\
LMM & $9.9412(0.7700) / 8.5-12.6$ \\
RMF & $11.036(0.9354) / 8.9-13$ \\
LMF & $11.092(0.9378) / 8.9-13$ \\
RMWC & $9.338(0.5367) / 7.9-10.3$ \\
LMWC & $9.196(0.8789) / 5.1-10.3$ \\
RMMC & $10.3841(0.9912) / 8.1-14.8$ \\
LMMC & $10.4021(0.9940) / 8.1 / 14.8$
\end{tabular}

$\mathrm{RMM}=$ right mandible mother, $\mathrm{LMM}=$ left mandible mother, $\mathrm{RMF}=$ right mandible, father, $\mathrm{LMF}=$ left mandible, father, $\mathrm{RMWC}=$ right mandible women controls, $\mathrm{LMWC}=$ left mandible women controls, $\mathrm{RMMC}=$ right mandible, men controls, LMMC = left mandible, men controls. 
Table 6. Comparison between right and left side of the controls' mandible.

\begin{tabular}{cc}
\hline Female and male controls & $\mathrm{p}$ \\
\hline RSFC vs LSFC & 0.1721 \\
RSMC vs LSMC & 0.2069 \\
\hline
\end{tabular}

RSFC $=$ right side female control; LSFC = left side female control; RSMC = right side male control; LSMC = left side male control.

[13], including the present one, the characteristics are very similar to those reported in the literature except in the family history of the patients. We thought this is probably because it is necessary to look for minor manifestations, for example the preauricular tags. We emphasized this to each patient to ask all members of their family. However, it could be that our population has more genetic variations and heritability with dominant pattern, more frequent than in other populations.

It was very interesting that we did not find differences between the measurements of the right and left sides of the mandible, neither cases nor controls, only in the patients' fathers $(p=0.0253)$, but when we compare the parents with the controls, we only observed differences in women. Anyway we found differences and we think it is probably a minimal expression of some variant present in parents, although this is controversial. We also expected we would find differences between family and non-family cases, but that did not happen. We assumed the family cases should be more predisposed to the malformation, but at least in the mandible area, it was not true.

On the other hand, in the measurement of the ears no differences were found between the cases and the controls, although the malformation of external ear is the most important clinical finding of the OAV syndrome [10].

There are not many studies that measure the mandible in a clinical way, the radiological measurement is almost always for orthodontic purposes and never in the parents to look for minimal clinical findings. However no cutoff point is described with exactitude, to say there is a pathological or normal difference between the measurements of both sides of the mandible. It is necessary to define this point and to compare with radiological studies, but we think the clinical measurements could be a good option to study the patients' parents and to establish if they have minor manifestations that could explain some heritability. It is very interesting that we found differences in the measurements of the mothers' mandible of the patients and their controls; in the mothers the mandible was bigger than in the controls, this probably means that the development of the cranial structures are modified by some genetic variants, more in women than in men; it is possible that the sex difference intervenes in the abnormal expression, resulting in bigger final dimensions in the patients' mothers than in their controls. The parents of the patients and their controls were matched by age, sex and all of them were Mexican mestizos and from the same geographical region to minimize the possible differences between them. 
It is very difficult to know exactly if the differences we found are important or not, especially with clinical measurements, however, as stated by Laster et al. even taking radiological measurements, there is no $100 \%$ accuracy to establish the diagnosis of abnormal facial asymmetry [11].

Also, it is important to say, there is facial asymmetry that is not secondary to hard tissues (bones), it depends on the soft tissues (muscles) [14]. In the OAV patients it could be secondary to both circumstances and different genes could be involved. We know that mandible morphogenesis results from the ectodermic and endodermic layer in the first place, but there are also mesodermal cores between the 2 previously mentioned layers [15].

It is also true that this study has another limitation because we only measure the mandibular body and we do not have the possibility, clinically, to measure the mandibular branches, which could be asymmetric. Recently, with 3D-CT images it is easier to find any asymmetry and more accurately. It could be a good idea to search some features in OAVS children's parents. [12]. The problem with these studies is the cost and the possibility of carrying them out in all institutions.

We do not know much about genetic basis of craniofacial anomalies, there is yet much to learn. Finally we can say there is too much missing but it is necessary to find some clinical tools, besides the molecular ones, to help us in genetic counseling.

\section{Conclusion}

It is very difficult to know exactly if the differences we found are important or not, especially with clinical measurements; however, even taking radiological measurements, there is no $100 \%$ accuracy in diagnosing facial asymmetry. The clinical measurements could be a good option to establish if the parents have minor manifestations that could explain some heritability.

\section{Conflicts of Interest}

The authors declare there is no conflict of interest.

\section{References}

[1] Barisic, I., Odak, L., Loane, M., et al. (2014) Prevalence, Prenatal Diagnosis and Clinical Features of Oculo-Auriculo-Vertebral Spectrum: A Registry-Based Study in Europe. European Journal of Human Genetics, 22, 1026-1033. https://doi.org/10.1038/ejhg.2013.287

[2] Poswillo, D. (1973) The Pathogenesis of the First and Second Branchial Arch Syndrome. Oral Surgery, 35, 302-327. https://doi.org/10.1016/0030-4220(73)90070-4

[3] Mastroiacovo, P., Corchia, C., Botto, L.D., et al. (1995) Epidemiology and Genetics of Microtia-Anotia: A Registry Based Study on over One Million Births. Journal of Medical Genetics, 32, 453-457. https://doi.org/10.1136/jmg.32.6.453

[4] Pegler, J.R., Soares, D.C., Quaio, C.R., et al. (2016) Clinical Description of 41 Brazilian Patients with Oculo-Auriculo-Vertebral Dysplasia. Revista da Associação 
Médica Brasileira, 62, 202-206. https://doi.org/10.1590/1806-9282.62.03.202

[5] Luquetti, D.V., Heike, C.L., Hing, A.V., Cunningham, M.L. and Cox, T.C. (2012) Microtia: Epidemiology and Genetics. American Journal of Medical Genetics Part $A, 158,124-139$. https://doi.org/10.1002/ajmg.a.34352

[6] Brotto, D., Manara, R., Ghiselli, S., et al. (2017) Oculo-Auriculo-Vertebral Spectrum; Going beyond the First and Second Pharyngeal Arch Involvement. Neuroradiology, 59, 305-3016. https://doi.org/10.1007/s00234-017-1833-Z

[7] Manara, R., Brotto, S., Ghiselli, R., et al. (2015) Cranial Nerve Abnormalities in Oculo-Auriculo-Vertebral Spectrum. American Journal of Neuroradiology, 36, 1375-1380. https://doi.org/10.3174/ajnr.A4273

[8] Beleza, A., Clayton, J., Saravia, M., et al. (2014) Oculo-Auriculo-Vertebral Spectrum: A Review of the Literature and Genetic Update. Journal of Medical Genetics, 51, 635-645. https://doi.org/10.1136/jmedgenet-2014-102476

[9] Muñoz-Pedroza, L. and Arenas-Sordo, M.L. (2013) Clinical Features of 149 Patients with Facio-Auriculo-Vertebral Spectrum. Acta Otorrinolaringologica Española, 64, 359-362. https://doi.org/10.1016/j.otorri.2013.03.007

[10] Alasti, F. and Van Camp, G. (2009) Genetics of Microtia and Associated Syndromes. Journal of Medical Genetics, 346, 361-369. https://doi.org/10.1136/jmg.2008.062158

[11] Laster, W.S., Ludlow, J.B., Bailey, L.J. and Hershey, H.G. (2005) Accuracy of Measurements of Mandibular Anatomy and Prediction of Asymmetry in Panoramic Radiographic Images. Dentomaxillofacial Radiology, 34, 343-349. https://doi.org/10.1259/dmfr/28020783

[12] Manara, R., Schifano, G., Brotto, D., Mardari, R., Ghiselli, S. and Gerunda, A. (2016) Facial Asymmetry Quantitative Evaluation in Oculo-Auriculo-Vertebral Spectrum. Clinical Oral Investigations, 20, 219-225.

https://doi.org/10.1007/s00784-015-1660-8

[13] Llano-Rivas, I., González-del Ángel, A., del Castillo, V., Reyes, R. and Carnevale, A. (1999) Microtia: A Clinical and Genetic Study at the National Institute of Pediatrics in Mexico City. Archives of Medical Research, 30, 120-124. https://doi.org/10.1016/S0188-0128(98)00023-2

[14] Nicot, R., Hottenstein, M., Raoul, G., et al. (2014) Nodal Pathway Genes Are Down-Regulated in Facial Asymmetry. Journal of Craniofacial Surgery, 25, e548-e555. https://doi.org/10.1097/SCS.0000000000001076

[15] Szabo-Rogers, H.L., Smithers, L.E., Yakob, W. and Liu, K.J. (2010) New Directions in Craniofacial Morphogenesis. Developmental Biology, 341, 84-94. https://doi.org/10.1016/j.ydbio.2009.11.021 